\title{
Family override for organ donation
}

\author{
Ian M. Ball, MD, MSc (1) • Robert Sibbald, MSc $\cdot$ Simon Oczkowski, MD, MSc
}

Received: 9 October 2019/Revised: 21 October 2019/Accepted: 22 October 2019/Published online: 26 November 2019

(C) Canadian Anesthesiologists' Society 2019

Consent for treatment in medicine is always preferable when obtained directly from patients, though increasingly in acute care situations clinicians obtain consent from surrogate decision makers. Consent for organ donation is unique in a number of aspects. For living donors, there is no direct medical benefit to them but they are exposed to a non-trivial risk. For deceased donation, donors are no longer our "patients", and thus we are not dealing with a "treatment" in the legal sense. These distinctions may leave physicians and substitute decision makers (SDMs) confused about their roles and to whom they are responsible. Doig et al. emphasized the importance of respecting "donor and family wishes,", 1,2 but little guidance exists for how to respond when these wishes are contradictory.

In this issue of Journal, Weiss et al. ${ }^{3}$ present the results of the first Canada-wide survey in over a decade of intensive care physicians' perspectives on the practice of organ donation. Their rigorously conducted survey focused on two controversial practices: family override of donation, and physician non-referral for organ donation of a potentially eligible patient. These two situations are of particular interest because they highlight the tension

\footnotetext{
I. M. Ball, MD, MSc ( ,

Division of Critical Care, Department of Medicine, Western University, London, ON, Canada

e-mail: Ian.Ball@lhsc.on.ca

R. Sibbald, MSc

London Health Sciences Center Ethics Program, Department of

Family Medicine, Western University, London, ON, Canada

S. Oczkowski, MD, MSc

Division of Critical Care, Department of Medicine, McMaster

University, Hamilton, ON, Canada
}

between duties owed to patients, to their families, and to the broader public.

Intensivists and organ donation organizations (ODO) occupy an unenviable and difficult position when faced with the knowledge that a patient would have wanted to donate their organs, but the SDM is overriding their wishes. The situation is clearly very common, as evidenced by the finding that $55 \%$ of survey respondents reported having witnessed family override situations. Only $2 \%$ of respondents stated they would continue to pursue a patient's expressed wish to donate in the face of family override, even though override does not appear to be consistent with legal recommendations ${ }^{1}$ or the strong ethical argument that family members ought not to interfere with the wishes of a patient when they are known. Why might well-meaning physicians undermine a patient's known wishes, and at the same time reduce the number of life-saving organs available for transplant? "Fear of loss of trust in the donation system" (81\%) and "obligation to respect the grief and desires of SDMs" (71\%) were cited as the two main reasons to respect family override requests. Other reasons cited by respondents included legal risks and additional stress for the physician. These sentiments are understandable, but tragic for patients as well as potential recipients.

Weiss et $a l .^{3}$ describe family override as "emotionally charged and ethically ambiguous." We wholeheartedly agree that these situations are "emotionally charged", but emotions should not drive practice. Instead, we postulate that the major driver is moral ambiguity. Healthcare providers have an ethical duty to protect their patients' autonomy. Where donation becomes murky is with regard to families-what are healthcare providers' obligations to them? While beneficent physicians should strive to do the most good for patients and families alike, should clinicians 
and ODOs spare family feelings at the cost of overriding patients' rights and societal interests? In living patients, there is no ambiguity. A physician's primary duty is to her/ his patient, not to their grieving family members, as callous as that may sound. What kind of duty physicians owe to their patients once they are dead is much less clear. It is ethically unclear what autonomy means for the deceased. The diversity of Canadians' beliefs and experiences may also add to the complexity of family override situations. The challenge for physicians, ODOs, and other healthcare team members is balancing individualism and collectivism. ${ }^{4}$ While on an individual basis the seemingly best option may be to "override the override," in the long run this might cause societal harm by creating mistrust in the donation system, a justification expressed by $81 \%$ of physician respondents. The principle of utilitarianism, ${ }^{5}$ optimizing the greatest good for the greatest number, would support physician respect of family override. As we are all taught in medical school, excellent communication (in this case with SDMs) is likely the best approach to addressing family requests for override of patients' expressed wishes to be organ donors, but it is not always effective.

There is no simple solution to this practical challengebut the status quo is not acceptable. The approach that Canadian ODOs have taken is to increase public awareness of organ donation, and we applaud their efforts. We suggest that better communication on the part of physicians and more widespread efforts are needed to improve next of kin's understanding of their responsibilities in these difficult situations. In our experience, powers of attorney for personal care often have little knowledge about their roles, or what the patients' wishes might be. This problem is not unique to donation, although conflicts involving patient wishes, family wants, and societal need are magnified in the donation arena.

The study by Weiss et al. has rekindled an extremely important discussion in the organ donation world. Solutions to this challenge will not come easily, but more dialogue seems like an important step in the right direction.

\section{La préséance de la volonté de la famille dans le don d'organes}

En médecine, il est toujours préférable d'obtenir le consentement au traitement directement du patient, mais il arrive de plus en plus fréquemment, dans des situations de soins aigus, que les cliniciens doivent obtenir le consentement d'un mandataire. Le consentement pour le don d'organes est particulier pour de nombreuses raisons. En cas de donneur vivant, le donneur ne retire aucun avantage médical de l'intervention tout en étant exposé à un risque loin d'être bénin. En cas de don après décès, les donneurs ne sont plus nos «patients », et nous n'avons donc pas affaire à un « traitement » au sens légal du terme. Ces distinctions peuvent créer la confusion tant chez les médecins que chez les mandataires quant à leurs rôles et à leurs loyautés. Doig et coll. ont souligné l'importance de respecter «les souhaits du donneur et de la famille », mais les lignes de conduite sont rares quant à l'attitude à adopter lorsque les souhaits de l'un et des autres sont contradictoires.

Dans ce numéro du Journal, Weiss et coll. ${ }^{3}$ présentent les résultats du premier sondage national canadien réalisé depuis plus d'une décennie sur les points de vue des intensivistes au sujet de la pratique du don d'organes. Les auteurs ont réalisé un sondage rigoureux en se concentrant sur deux pratiques controversées: la préséance de la volonté de la famille sur le don, et la non-référence médicale pour don d'organes d'un patient potentiellement éligible. Ces deux situations sont particulièrement intéressantes parce qu'elles mettent en lumière la tension entre les obligations envers nos patients, leurs familles, et le grand public.

Les intensivistes et les organismes de dons d'organes (ODO) sont dans une position peu enviable et difficile lorsqu'ils sont confrontés à une situation dans laquelle ils savent qu'un patient aurait voulu donner ses organes, mais que le mandataire va à l'encontre de ses volontés. La situation est assurément très courante, comme le montre le résultat selon lequel $55 \%$ des répondants au sondage rapportaient avoir été témoins de situations dans lesquelles la décision de la famille allait à l'encontre de celle du patient. Seuls $2 \%$ des répondants ont déclaré qu'ils continueraient à aller de l'avant avec le vœu exprimé d'un patient de faire don de ses organes malgré le refus de la famille, même si la préséance de la volonté de la famille ne semble pas coïncider avec les recommandations légales ${ }^{1}$ ou le fort argument déontologique selon lequel la famille ne devrait pas interférer avec les volontés d'un patient lorsqu'elles sont connues. Pourquoi est-ce que des médecins, forts de leurs bonnes intentions, iraient à l'encontre des volontés connues d'un patient, tout en réduisant le nombre d'organes vitaux disponibles pour la greffe? «La peur d'une perte de confiance dans le système de don » $(81 \%)$ et « l'obligation de respecter le deuil et les souhaits des mandataires » $(71 \%)$ sont les deux raisons principales citées de respecter les demandes de préséance de la famille. Parmi les autres raisons invoquées par les répondants figuraient les risques légaux et le stress supplémentaire pour le médecin. Ces sentiments sont 
certes compréhensibles, mais ils sont tragiques pour les patients aussi bien que pour les récipiendaires potentiels.

Weiss et coll. ${ }^{3}$ décrivent la préséance de la volonté de la famille comme étant «tendue émotionnellement et ambiguë d'un point de vue déontologique. » Nous sommes tout à fait d'accord sur le fait que ces situations sont «tendues émotionnellement », mais les émotions ne devraient pas être le moteur de notre pratique. Au lieu de cela, nous postulons que l'incitatif majeur réside dans l'ambiguïté morale. Les fournisseurs de soins de santé ont un devoir déontologique de protéger l'autonomie de leurs patients. Le don d'organes devient plus nébuleux lorsqu'il est question des familles de nos patients : quelles sont les obligations des fournisseurs de soins de santé envers elles? Alors que des médecins bienfaisants devraient s'efforcer de faire le plus de bien pour les patients et pour leurs familles, les cliniciens et les ODO devraient-ils épargner les sentiments d'une famille en faisant fi des droits des patients et des intérêts sociétaux? Pour un patient en vie, il n'y a aucune ambiguïté. L'obligation première d'un médecin est envers son patient, et non envers les membres endeuillés de la famille, même si cela semble cruel. Quel genre d'obligations ont les médecins envers leurs patients une fois ces derniers décédés? La réponse à cette question est bien moins claire. D'un point de vue déontologique, lorsqu'il s'agit d'un patient décédé, qu'entend-on par autonomie? La diversité des croyances et des expériences des Canadiens vient également ajouter à la complexité des situations de préséance de la volonté de la famille. Pour les médecins, les ODO et les autres membres de l'équipe de soins de santé, le défi est de trouver un équilibre entre individualisme et collectivisme. ${ }^{4}$ Alors que, d'un point de vue individuel, l'option vraisemblablement meilleure consiste à «ignorer la préséance », cela pourrait, à long terme, causer des torts à la société en sapant la confiance du public dans le système de don d'organes - une justification citée par $81 \%$ des médecins répondants. Le principe d'utilitarisme, ${ }^{5}$ qui consiste à maximiser le plus grand bien pour le plus grand nombre, appuierait le respect du médecin de la préséance de la famille. Comme nous l'avons tous appris pendant nos études de médecine, une communication ouverte (dans ce cas avec les mandataires) constitue probablement la meilleure approche pour discuter des demandes de la famille d'aller à l'encontre des volontés exprimées par un patient de faire don de ses organes, mais ce n'est pas toujours efficace.

Il n'existe pas de solution simple à ce défi pratique mais le statu quo n'est pas acceptable. L'approche adoptée par les ODO canadiens est d'augmenter la prise de conscience du public face aux dons d'organes, et nous louons leurs efforts. Nous suggérons qu'une meilleure communication de la part des médecins et des efforts plus soutenus sont nécessaires pour améliorer la compréhension des parents proches de leurs responsabilités dans ces situations délicates. Selon notre expérience, les mandataires légaux en regard des soins personnels ne connaissent souvent que bien peu leurs rôles - ou les volontés du patient. Certes, ce problème ne touche pas exclusivement la question du don, mais les conflits portant sur les volontés du patient, les désirs de la famille et les besoins sociétaux sont amplifiés lorsqu'on parle de don d'organes.

L'étude de Weiss et coll. a ravivé une discussion extrêmement importante dans le monde du don d'organes. Les solutions à ce défi ne seront pas faciles à trouver, mais le dialogue semble être un pas important dans la bonne direction.

\section{Conflicts of interest None.}

Funding statement None.

Editorial responsibility This submission was handled by Dr. Sangeeta Mehta, Associate Editor, Canadian Journal of Anesthesia.

Conflit d'intérêt Aucun.

Déclaration de financement Aucune.

Responsabilit' 'ditoriale Cet article a été traité par Dre Sangeeta Mehta, rédactrice adjointe, Journal canadien d'anesthésie.

\section{References}

1. Doig CJ, Rocker G. Retrieving organs from non-heart beating organ donors: a review of medical and ethical issues. Can J Anesth 2003; 50: 1069-76.

2. Institute of Medicine. Non-Heart-Beating Organ Transplantation: Medical and Ethical Issues in Procurement. Washington DC: National Academy Press; 1997 .

3. Weiss MJ, English SW, D'Aragon F, et al. Survey of Canadian intensivists on physician non-referral and family override of deceased organ donation. Can J Anesth 2020; DOI: https://doi.org/ 10.1007/s12630-019-01538-x.

4. Toews M, Caulfield T. Evaluating the "family veto" of consent for organ donation. CMAJ 2016; 188: E436-7.

5. Mack $P$. Utilitarian ethics in healthcare. International Journal of the Computer, the Internet and Management 2004; 12: 63-72.

Publisher's Note Springer Nature remains neutral with regard to jurisdictional claims in published maps and institutional affiliations. 